\title{
Immunoreactivity and a new staining method of monocarboxylate transporter 1 located in endothelial cells of cerebral vessels of human brain in distinguishing cere- bral venules from arterioles
}

\author{
Yuan Cao, ${ }^{1}$ Dong-Hui Ao, ${ }^{1}$ Chao Ma, ${ }^{2}$ Wen-Ying Qiu, ${ }^{2 *}$ Yi-Cheng Zhu ${ }^{1 *}$ \\ ${ }^{1}$ Department of Neurology, State Key Laboratory of Complex Severe and Rare Diseases, Peking Union Medical College \\ Hospital, Chinese Academy of Medical Sciences and Peking Union Medical College, Beijing \\ ${ }^{2}$ Department of Human Anatomy, Histology and Embryology, Institute of Basic Medical Sciences, Neuroscience \\ Center, Chinese Academy of Medical Sciences, School of Basic Medicine, Peking Union Medical College, Beijing, \\ China \\ *These authors contributed equally.
}

\begin{abstract}
Distinguishing brain venules from arterioles with arteriolosclerosis is less reliable using traditional staining methods. We aimed to immunohistochemically assess the monocarboxylate transporter 1 (MCT1), a specific marker of venous endothelium found in rodent studies, in different caliber vessels in human brains. Both largeand small-caliber cerebral vessels were dissected from four autopsy donors. Immunoreactivity for MCT1 was examined in all autopsied human brain tissues, and then each vessel was identified by neuropathologists using hematoxylin and eosin stain, the Verhoeff's Van Gieson stain, immunohistochemical stain with antibodies for $\alpha$-smooth muscle actin and MCT1 in sequence. A total of 61 cerebral vessels, including 29 arteries and 32 veins were assessed. Immunoreactivity for MCT1 was observed in the endothelial cells of various caliber veins as well as the capillaries, whereas that was immunenegative in the endothelium of arteries. The different labeling patterns for MCT1 could aid in distinguishing various caliber veins from arteries, whereas assessment using the vessel shape, the internal elastic lamina, and the pattern of smooth muscle fibers failed to make the distinction between small-caliber veins and sclerotic arterioles. In conclusion, MCT1 immunohistochemical staining is a sensitive and reliable method to distinguish cerebral veins from arteries.
\end{abstract}

Key words: Monocarboxylate transporter 1; venule; arteriole; human neuropathology.

Correspondence: Yi-Cheng Zhu, State Key Laboratory of Complex Severe and Rare Diseases, Peking Union Medical College Hospital, Peking Union Medical College and Chinese Academy of Medical Science, No. 1 Shuaifuyuan, Wangfujing, Beijing 100730, China. Tel. +86.010.69154059. E-mail: zhuych910@163.com

Contributions: YCZ, WYQ, CM, conception and design; YCZ, administrative support; WYQ, CM, provision of study materials or patients; YC, DHA, collection and assembly of data; YC, data analysis and interpretation. All the authors contributed to manuscript drafting, have read and approved the final version of the manuscript and agreed to be accountable for all aspects of the work in ensuring that questions related to the accuracy or integrity of any part of the work are appropriately investigated and resolved.

Conflict of interest: The authors have no conflicts of interest to declare, and all authors confirm accuracy.

Funding: This work was supported by the National Natural Science Foundation of China (No. 81971138).

Ethical Approval and consent to participate: The study was approved by the Institutional Review Board of the Institute of Basic Medical Sciences, Chinese Academy of Medical Sciences (Approval Number: 009-2014) and informed consent was taken from all individual participants. 


\section{Introduction}

There is accumulating evidence suggesting that cerebral venule disease might relate to leukoaraiosis and Alzheimer's disease, most of which come from neuroimaging studies. ${ }^{1-7}$ Although in pressing need, neuropathological studies on venules are scarce, likely owing to limited histological methods in identifying cerebral venules. Large-caliber cerebral arteries and veins can be histologically differentiated by the vessel shape and the presence of an internal elastic lamina, which is infeasible in small vessels. ${ }^{8}$

To date, there are two methods proposed for small-caliber arteriovenous identification in human histopathological studies. Staining for alkaline phosphatase was firstly proposed in 1995 by Moody and colleagues, yet the time-critical sampling procedure to maintain alkaline phosphatase activity limited the application of this method. ${ }^{1}$ The other method is anti- $\alpha$-smooth muscle actin ( $\alpha$ SMA) immunohistochemical staining, which relies on the characteristic pattern of the continuous wreath of concentric smooth muscle in the arteries. ${ }^{9}$ However, arteriole may be misinterpreted as venule when arteriolosclerosis occurs, since arteriolosclerosis manifests as loss of smooth muscle cells and deposits of fibrohyalinized material. ${ }^{10}$

Various hierarchical signaling proteins are involved in the early and late stages of arteriovenous differentiation and vascular development, and result in expressing of specific molecules in the endothelial cells of arteries or veins. ${ }^{11}$ Recently, Michael et al. revealed the molecular definitions of vascular arteriovenous types using single-cell transcriptomics in adult mice, and identified that monocarboxylate transporter 1 (MCT1), encoded by solute carrier 16a1 gene (Slc16al), is an underlying endothelial venous and capillary marker, whereas it remains to be clarified in human brains. ${ }^{12}$ In the current study, we aim to examine the immunoreactivity for MCT1 in various caliber vessels and explore whether MCT1 immunostaining is a sensitive and reliable method to discriminate between venules and arterioles in human brains.

\section{Materials and Methods}

All brain tissue samples were obtained at autopsy from four donors of the National Human Brain Bank for Development and Function (http://anatomy.sbm.pumc.edu.cn/brainbank), located at the Chinese Academy of Medical Sciences/Peking Union Medical College (CAMS/PUMC). Four cases without a history of cere- brovascular disease were included and the main characteristics were shown in Supplementary Table 1. The post-mortem delay of brain tissues was within 24 hours. The research protocol was approved by the Institutional Review Board of the Institute of Basic Medical Sciences of the CAMS/PUMC, Beijing, China (Approval Number: 009-2014), and informed consent was obtained from all individual participants. A detailed protocol describing the collection and processing was published elsewhere. ${ }^{13,14}$ According to the National Institute of Aging and the Alzheimer's Association (NIA-AA) guidelines, all vessels dissected from brain tissue samples were embedded in conventional paraffin after gross examination and sectioned at a 5 - $\mu \mathrm{m}$ thickness.

Cerebral vascular tissues were divided into large-caliber and small-caliber vessels. Large-caliber vessels include common carotid arteries, internal carotid arteries, and middle cerebral arteries which respectively represented elastic arteries and muscular arteries, as well as corresponding veins such as internal jugular veins, great cerebral veins of Galen, and internal cerebral veins. Small-caliber vessels include small arteries and veins, which were defined as vessels visible under microscopy without anatomical names, normally larger than $300 \mu \mathrm{m}$ or $200 \mu \mathrm{m}$ and less than $1 \mathrm{~mm}$ in diameter, as well as arterioles and venules were defined as vessels having caliber less than $300 \mu \mathrm{m}$ or $200 \mu \mathrm{m}$ in diameter. ${ }^{15}$ All small-caliber cerebral vessels were detected in the frontal white matter adjacent to the anterior horn of the lateral ventricles, caudate/putamen, and occipital white matter adjacent to the posterior horn of the lateral ventricles.

Firstly, immunohistochemical examination for anti-MCT1 (Proteintech Cat\# 20139-1-AP, RRID: AB_2878645, rabbit polyclonal antibody, diluted 1:800) receptor was performed in all slides. It was replicated in another consecutive slice to verify the immunoreactivity and negative control was also carried out. Secondly, for identifying the vessel type, hematoxylin and eosin (H\&E), Verhoeff's Van Gieson (VVG), immunohistochemistry against aSMA (Cat\# 19245, RRID: AB_2734735, rabbit monoclonal antibody, diluted 1:400; Cell Signaling Technology, Danvers, MA, USA) and MCT1 were conducted on the prepared unstained sections. For double immunofluorescence, MCT1, aSMA and vascular endothelial growth factor C (VEGF-C, Cat\# 22601-1-AP, RRID: AB_2879132, rabbit polyclonal antibody, diluted 1:200; Proteintech, Rosemont, IL, USA) antibodies were incubated with the CD31 (Cat\# ab9498, RRID: AB_307284, mouse monoclonal antibody, diluted 1:200; Abcam, Cambridge, UK) antibody, respectively. The sites of antigen-antibody reactions were detected by using AlexaFluor 488-labeled anti-mouse IgG

Table 1. The basic characteristics of cerebral vessels classified by structure and diameter of vessel walls.

\begin{tabular}{|c|c|c|c|c|c|c|c|}
\hline Groups & n & Detected vessels & Average diameters & V & & aSMA & MCT1 \\
\hline & & & $($ mean \pm SD) & Elastic fibers & Collagen fibers & & \\
\hline Elastic arteries & $n=10$ & Common carotid artery & $6.28 \pm 1.63 \mathrm{~mm}$ & ++ & + & ++ & - \\
\hline & & Internal carotid artery & $4.75 \pm 1.12 \mathrm{~mm}$ & ++ & + & ++ & - \\
\hline Muscular arteries & $\mathrm{n}=7$ & Middle cerebral artery & $2.7 \pm 0.88 \mathrm{~mm}$ & + & + & +++ & - \\
\hline Small arteries* & $\mathrm{n}=4$ & Small arteries on 3 selected regions & $0.88 \pm 0.32 \mathrm{~mm}$ & + & ++ & + & - \\
\hline Arterioles* & $n=8$ & Arterioles on 3 selected regions & $123.86 \pm 36.72 \mathrm{~m}$ & - & ++ & + & - \\
\hline Venules* & $\mathrm{n}=8$ & Venules on 3 selected regions & $182.37 \pm 58.49 \mathrm{~m}$ & - & ++ & + & + \\
\hline Small veins* & $\mathrm{n}=4$ & Small veins on 3 selected regions & $0.72 \pm 0.28 \mathrm{~mm}$ & - & ++ & + & + \\
\hline Medium caliber veins & $\mathrm{n}=6$ & Internal cerebral vein & $0.78 \pm 0.22 \mathrm{~mm}$ & - & + & $+/-$ & + \\
\hline Large caliber veins & $\mathrm{n}=14$ & Great cerebral vein of Galen & $1.53 \pm 0.48 \mathrm{~mm}$ & - & + & + & + \\
\hline & & Internal jugular vein & $7.74 \pm 2.02 \mathrm{~mm}$ & $+/-$ & + & $+/-$ & + \\
\hline
\end{tabular}

VVG, Verhoeff's Van Gieson; SMA, smooth muscular actin; MCT1, monocarboxylate transporter 1; *the selected regions include frontal white matter adjacent to anterior horn of the lateral ventricles, caudate/putamen, and occipital white matter adjacent to posterior horn of the lateral ventricles. 
(Cat\# A-11001, RRID: AB_2534069, diluted 1:500; Thermo Fisher Scientific, Waltham, MA, USA) and 647-labeled anti-rabbit IgG (Cat\# A-21244, RRID: AB_2535812, diluted 1:500; Thermo Fisher Scientific). The Masson trichrome stain was also conducted in small-caliber vessels to assess the degree of collagen deposition. For H\&E-stained slides, criteria used to distinguish the venous system from the arterial system included the following: veins and venules are oval or oblong and collapse easily; arteries and arterioles are round, and the lumens are rigid. The elastic fibers stained black, and collagen stained red by VVG, and vessels were classified based on the presence or absence of an internal elastic lamina. ${ }^{8}$ For $\alpha$ SMA slides, the artery was defined by a continuous wreath of concentric smooth muscle, and the vein was defined by bundled smooth muscle fibers with intermixed collagen. ${ }^{8,9}$ For MCT1 stain-
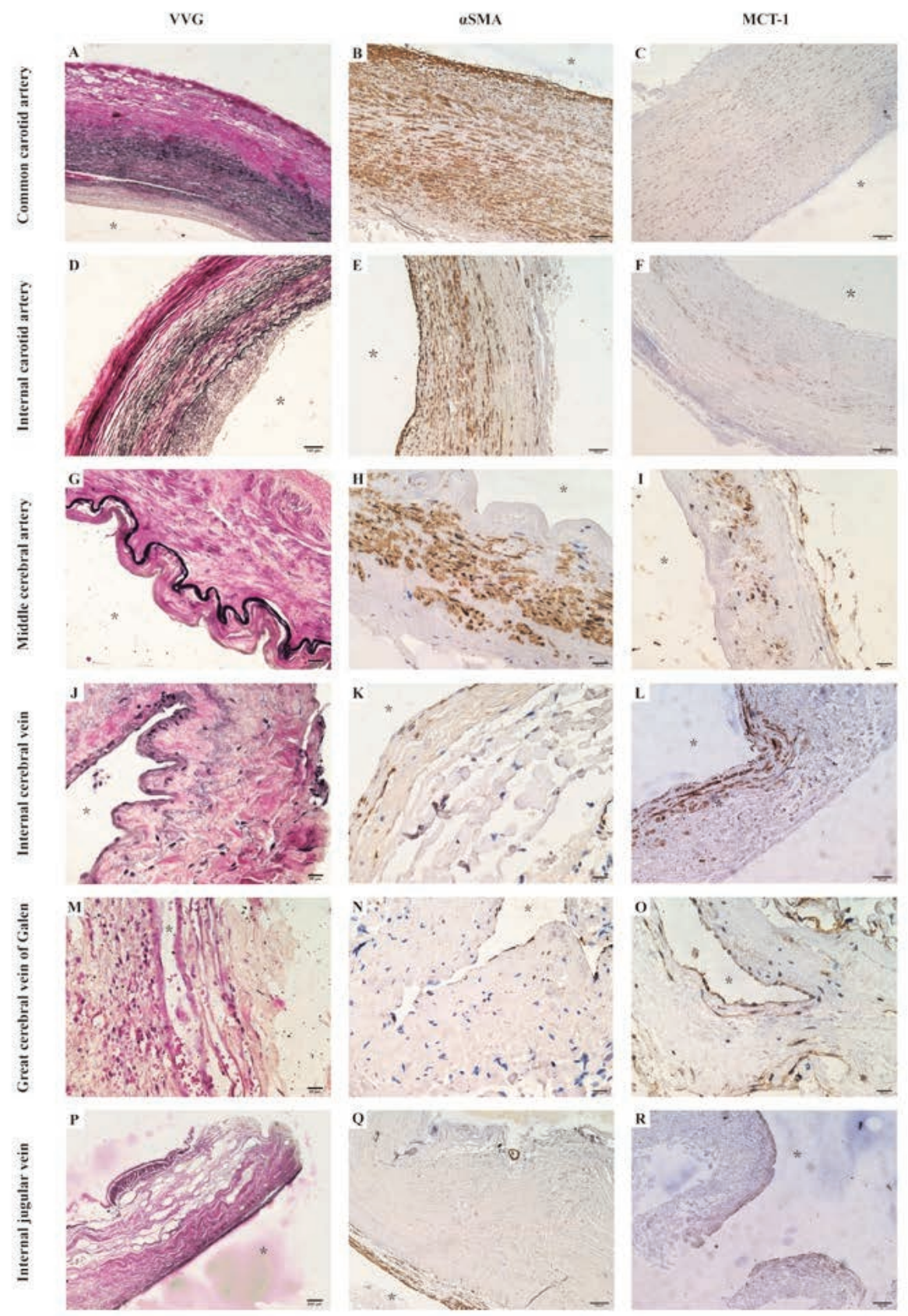

Figure 1. Different patterns of elastic fibers, smooth muscle cells, and monocarboxylate transporter 1 in arteries and veins. The Verhoeff's Van Gieson (VVG)-stained section showed that the abundant elastic fibers were demonstrated significantly in the common carotid artery (A) and the internal carotid artery (D), and the presence of the internal elastic lamina in the middle cerebral artery (G) was still predominantly which was completely different from veins without an elastic lamina (J,M,P). Anti-alpha-smooth muscle actin ( $\alpha$ SMA) immunohistochemistry showed that the smooth muscle pattern in the arteries was demonstrated by a continuous wreath of concentric smooth muscle (B,E,H), while for veins, there was less bundled smooth muscle demonstrated in walls (K,N,Q). Monocarboxylate transporter 1 (MCT1) was immunopositive in the venous endothelium using anti-MCT1 immunohistochemistry $(\mathrm{L}, \mathrm{O}, \mathrm{R})$, while the endothelial cells of arteries were non-stained despite some modest stains were demonstrated in the media tunica (C, F, and I). Scale bars: A,P) $200 \mu \mathrm{m}$; B-F,L,Q,R) $100 \mu \mathrm{m}$; G-I,J,K,M-O) $20 \mu \mathrm{m}$. 
ing slides, the assessment of arteriovenous identification is based on the absence or presence of MCT1 stain in the endothelium.

\section{Results}

A total of 61 vessels were detected, including 10 elastic arteries, 7 muscular arteries, 4 small arteries, 8 arterioles, 20 large-caliber veins, 4 small veins and 8 venules. Characteristics of each vessel group are described in Table 1.

\section{Immunoreactivity for MCT1 in various cerebral vessels}

Using immunohistochemistry, we assessed the distribution of
MCT1 in both arteries and veins. All of the cerebral venous slides revealed intense labeling for MCT1 in endothelium regions (Figure $1 \mathrm{~L}, \mathrm{O}, \mathrm{R}$ and Figure $2 \mathrm{H}, \mathrm{P}$ ). A similar but less intense labeling pattern was seen in the media tunica of some arteries, whereas all arterial endothelium was not stained. (Figure 1 C,F,I) Staining for MCT1 was also observed in microvessels such as capillaries and postcapillary venules.

\section{Vessel type identification}

For large-caliber cerebral vessels, the shape in the cross-section and smooth muscle pattern was consistent with previous studies, and also the internal elastic lamina was different in arteries and veins (Figure 1). For the elastic artery, in addition to the round walls, the wreath of smooth muscle and abundant elastic fibers in
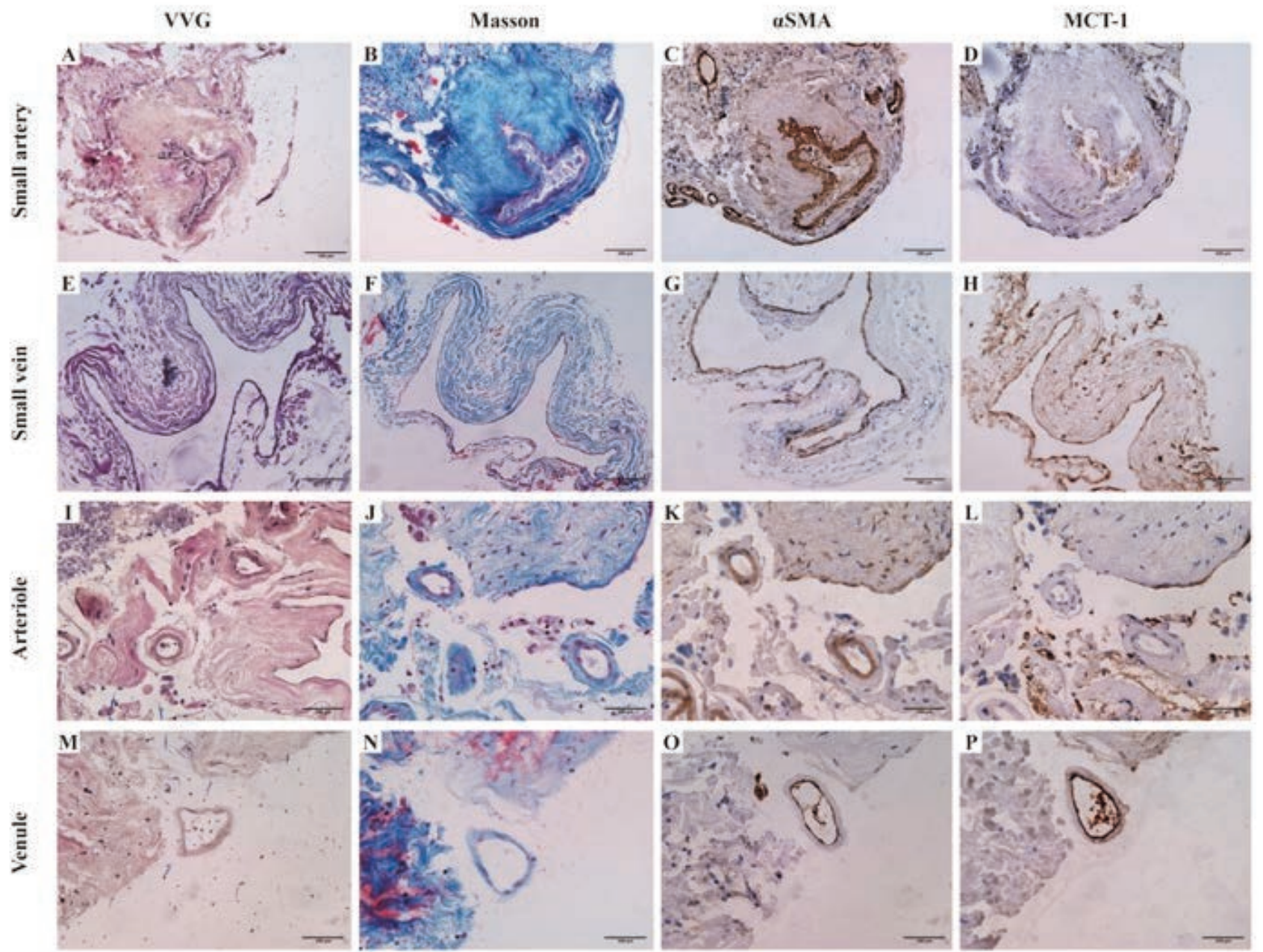

Figure 2. Verhoeff's Van Gieson (VVG) staining (A,E,I,M); Masson's trichrome staining (B,F,J,N); anti-alpha-smooth muscle actin ( $\alpha$ SMA) immunohistochemistry $(\mathrm{C}, \mathrm{G}, \mathrm{K}, \mathrm{O})$ and anti-monocarboxylate transporter 1 (MCT1) immunohistochemistry (D,H,L,P) in small-caliber cerebral vessels. The internal elastic lamina was still present in most small arteries (A), while it became disappeared in arterioles $(<200 \mu \mathrm{m}, \mathrm{I})$ which was similar to veins and venules $(<300 \mu \mathrm{m}, \mathrm{E}, \mathrm{M})$. Masson's trichrome staining demonstrated the collagen fibers deposition existed to different extents $(\mathrm{B}, \mathrm{F}, \mathrm{J}, \mathrm{N})$. Little difference in the smooth muscle pattern existed between small-caliber arteries and veins, although the smooth muscle cells in small arteries $(\mathrm{C})$ and arterioles $(\mathrm{K})$ were more thickened than that in small veins $(\mathrm{G})$ and venules $(\mathrm{O})$, respectively. MCT1 was still immunopositive in the endothelium of small veins $(\mathrm{H})$ and venules $(\mathrm{P})$, while the endothelial cells of small arteries (D) and arterioles (P) were non-stained, suggesting consistent and stable discrimination. Scale bars: $100 \mu \mathrm{m}$. 
the tunica media were notably demonstrated. Like the elastic artery, the muscular artery also has the same shape and smooth muscle pattern, while only the internal elastic lamina and much lesser elastic fibers in the tunica media were observed. Large-caliber veins demonstrated an oval or collapsed shape in cross-section, bundled smooth muscle cells, and a lack of the internal elastic lamina. Immunohistochemical examination using the antibody for MCT1 showed immunoreactivity in all venous endothelial cells, which showed a distinctive pattern to differentiate a vein from an artery.

All small-caliber vessels have no distinctive internal elastic lamina and have no notable differences in smooth muscle pattern, especially for arterioles with severe fibrohyalinization. The crosssectional shape of small veins and venules collapsed easily, while that of small arteries and arterioles was not as round and rigid as large-caliber arteries. However, there are obvious distinctions of MCT1 between small-caliber arteries and veins (Figure 2). The $\mathrm{CD} 31^{+} / \mathrm{MCT}^{-}{ }^{-}$small vessel is considered as an arteriole with positively immunolabeled for $\alpha$ SMA in the media tunica and VEGF$\mathrm{C}$ in the intima tunica (Figure 3). The considered venule demonstrated a co-localized pattern for both CD31 and MCT1 in the endothelium, whereas negative to the aSMA and VEGF-C immunostaining.

For fibrohyalinized arterioles and collagenized venules, identification based on smooth muscle cells and collagen without an absolute cross-section was not feasible (Figure 4). The slides lacked a typical cross-section shape, an internal elastic lamina, and smooth muscle pattern, whereas demonstrated an MCT1 immunopositivity which indicated it was a venule rather than an arteriole.

\section{Discussion}

In this study, immunohistochemical findings of MCT1 receptors on different caliber cerebral vessels were first been represented in human brains. Our findings suggested that the immunoreactivity for MCT1 was negative on the endothelium of both large and small caliber arteries, whereas it was prominently apparent in various venous endothelium, especially for small veins and venules. Given this characteristic, evaluation based on endothelial MCT1 immunostaining is reliable to determine artery and vein in human brains.

It is known that MCT1, also named solute carrier 16a1 (SLC16A1), is an important membrane transport protein mediating the translocation of monocarboxylates. ${ }^{16,17}$ Previous studies have already detected a consistent immunoreactivity for MCT1 in cerebral vessels of both adult human and rodent brains, while its distribution pattern in arteries and veins remained unexplored. ${ }^{18-20}$ Single-cell transcriptomics profiled the principal cell types of the mice brain vasculature, which clarified that the expression of Slc16al transcripts and the corresponding protein MCT1 were specific to capillary-venous zonation. ${ }^{12}$ Our study further verified that MCT1 immunostaining can also display positive in adult human cerebral venous endothelium as well as capillaries, which may due to the role of capillaries, postcapillary venules, and veins in regulating the acidity by excreting out large amounts of lactic acids with the collaboration of MCT $1 .{ }^{18}$ In addition, we also observed MCT1 immunopositivity in the tunica media of large-caliber arteries, which have not been reported before. The prior pathological finding from pulmonary arterial hypertension patients indicated that the elevation of lactate was observed in pulmonary artery smooth muscle cells, which may stimulate MCT1 expression in smooth muscle cells with vascular remodeling. ${ }^{21}$ Accordingly, a similar mechanism of vascular remodeling and smooth muscular
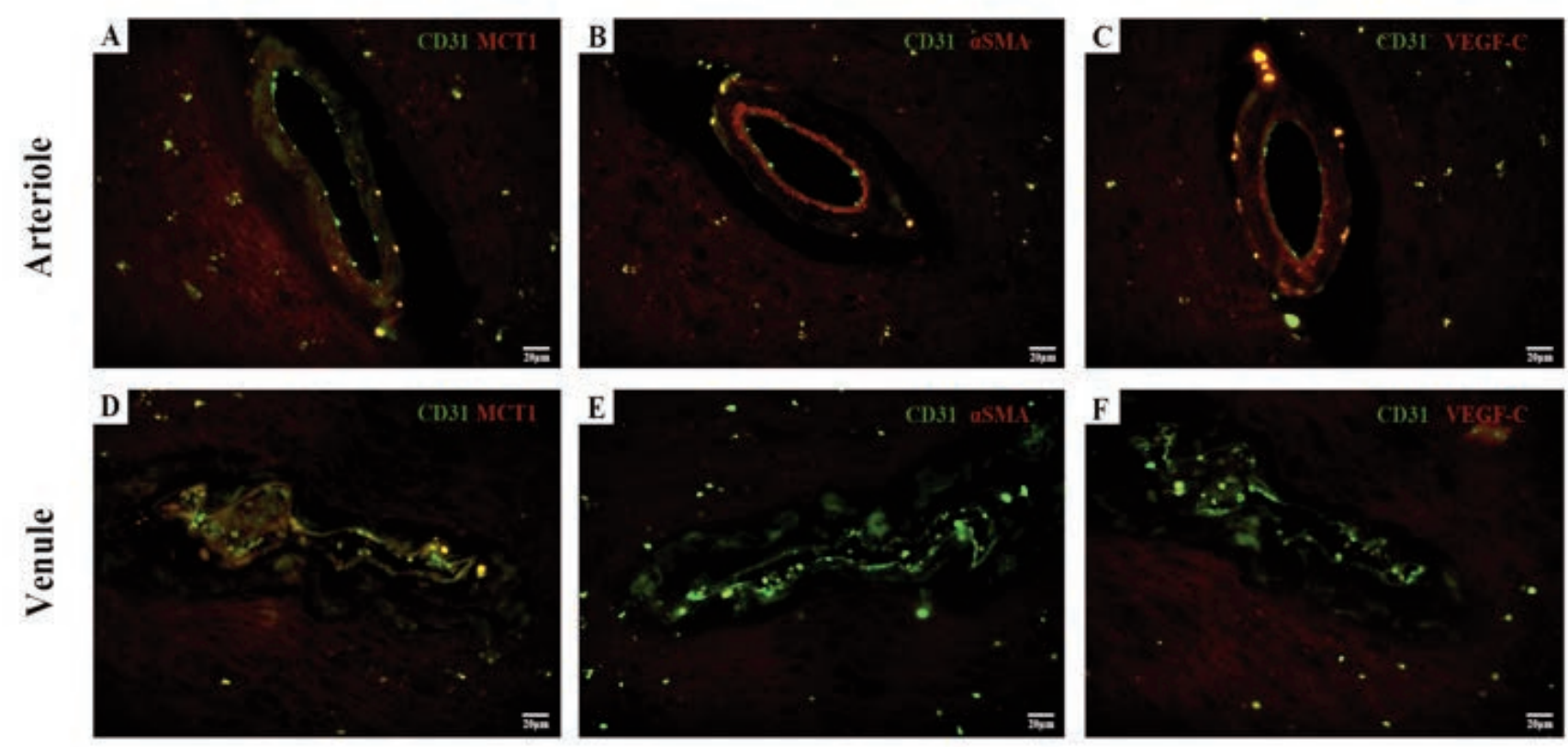

Figure 3. Immunofluorescence staining of small vessel sections. The endothelium is immunolabeled with CD31. The first CD31immunolabeled vessel is considered as an arteriole with a negative immunoreactivity for MCT1 (A) along endothelium but positive for both XSMA (B) in the media tunica and VEGF-C (C) in the intima tunica. The second vessel is considered as a venule with the double-staining for CD31 and MCT1 (D) in endothelial cells, but immune-negative for $\alpha$ SMA (E) and VEGF-C (F). Scale bars: $20 \mu \mathrm{m}$. 

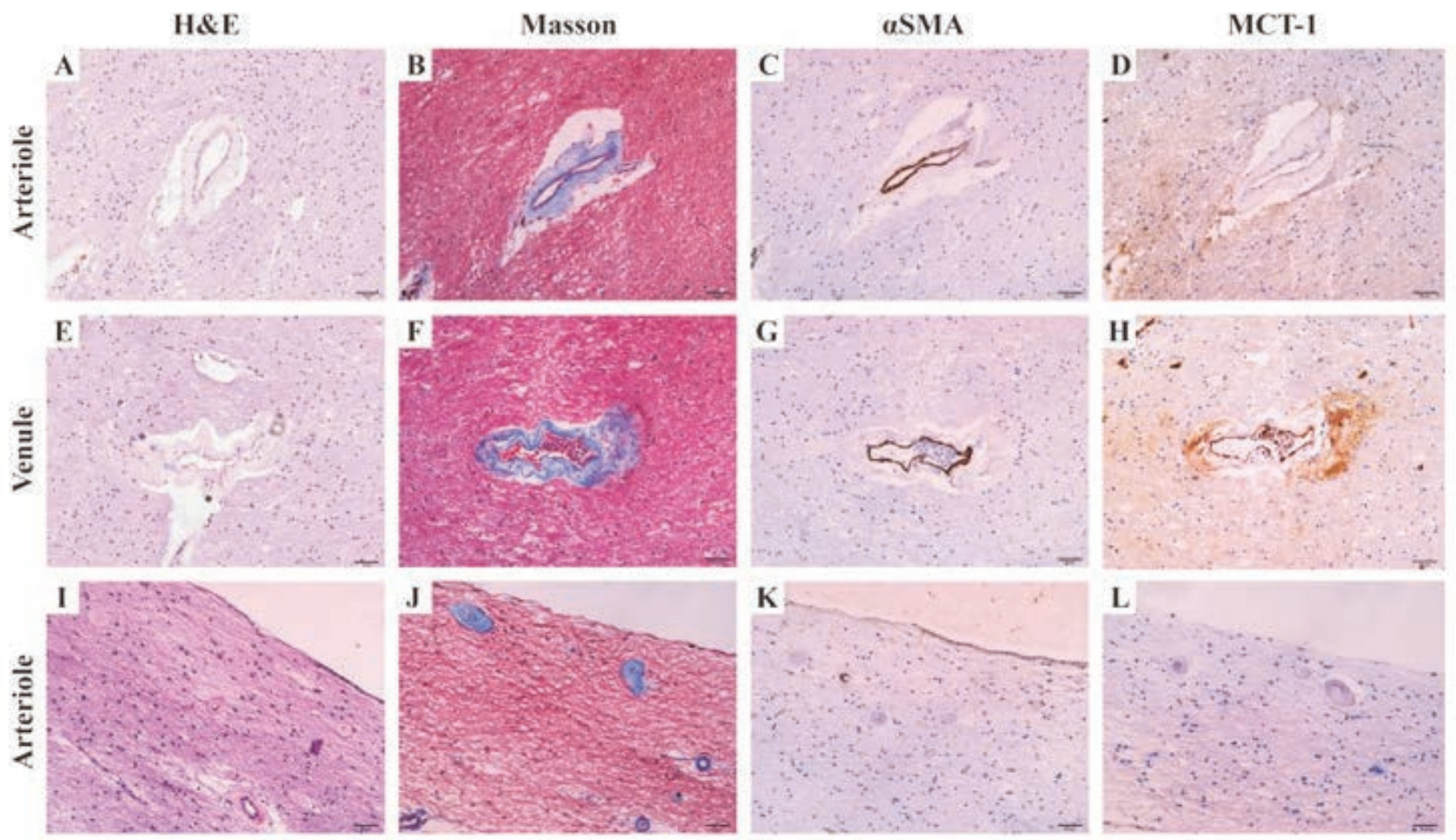

Figure 4. The hematoxylin and eosin (H\&E) staining (A,E,I), Masson's trichrome staining (B,F,J), anti-alpha-smooth muscle actin ( $Q S M A)$ immunohistochemistry $(\mathrm{C}, \mathrm{G}, \mathrm{K})$, and anti-monocarboxylate transporter 1 (MCT1) immunohistochemistry (D,H,L) in discordant cases. The arteriole (A-D) without a typical cross-section shape was not as round and rigid as the artery (A), and Masson's trichrome staining (B) demonstrated collagen fibers deposition which indicated fibrohyalinized change leading to thinner smooth muscle cells than normal (C). Collagenosis also affected the venule (E,F), while the immunoreactivity of MCT1 in endothelium was still visible clearly $(\mathrm{H})$. For severe fibrohyalinized arterioles $(\mathrm{I}, \mathrm{J})$, even some leading to a stenosis of the vessel lumen, failed to stain with anti- $\alpha$ SMA (K) while MCT1 negative $(\mathrm{L})$ indicated that these were arterioles $(\mathrm{J})$. In this case, classification based on the smooth muscle cells to distinguish arterioles from venules did not work. Scale bars: $50 \mu \mathrm{m}$.

proliferation may be also performed in the large-caliber cerebral vessels and contribute to MCT1 expression upregulation.

For large-caliber cerebral vessels, arteriovenous type can be identified by the shape, the internal elastic lamina, and the pattern of smooth muscle cells. The immunoreactivity of MCT1 observed in the venous endothelium and some labeling in arterial tunica media can also make an accurate distinguishment of vascular type. For small-caliber cerebral vessels, previous histopathological studies may struggle to distinguish between a collagenized $<150 \mu \mathrm{m}$ cerebral vein and a small artery with arteriolosclerosis using the Masson trichrome stain and anti- $\alpha$ SMA immunostaining. ${ }^{9}$ Indeed, they might misinterpret the periventricular sclerotic arterioles as collagenized venules due to the loss of smooth muscle cells in tunica media layers. The steady anti-MCT1 immunostaining on venous endothelium rather than artery endothelium provides a reliable method in arteriovenous discrimination, regardless of the presence of arteriolosclerosis or venule collagenosis. Given the importance of identifying these pathological changes in studies, the immunohistochemical staining method with MCT1 antibodies is of value. It is to note that the anti-MCT1 immunostaining is inaccurate to make a distinction between postcapillary venules and capillaries because the structure of their walls and the physiological function are almost identical.

Our findings reported the distinctive immunoreactivity for MCT1 on endothelial cells of human cerebral vessels from that of arteries, and further verified that MCT1 immunohistochemical staining is a sensitive and reliable method to distinguish cerebral veins from arteries.

\section{References}

1. Moody DM, Brown WR, Challa VR, Anderson RL. Periventricular venous collagenosis: association with leukoaraiosis. Radiology 1995;194:469-76.

2. Klakotskaia D, Agca C, Richardson RA, Stopa EG, Schachtman TR, Agca Y. Memory deficiency, cerebral amyloid angiopathy, and amyloid- $\beta$ plaques in APP+PS1 double transgenic rat model of Alzheimer's disease. PLoS One 2018; 13:e0195469.

3. Brown WR, Thore CR. Review: cerebral microvascular pathology in ageing and neurodegeneration. Neuropathol Appl Neurobiol 2011;37:56-74.

4. Pettersen JA, Keith J, Gao F, Spence JD, Black SE. CADASIL accelerated by acute hypotension: Arterial and venous contribution to leukoaraiosis. Neurology 2017;88:1077-80.

5. Bouvy WH, Kuijf HJ, Zwanenburg JJ, Koek HL, Kappelle LJ, Luijten PR, et al. Abnormalities of cerebral deep medullary veins on 7 Tesla MRI in amnestic mild cognitive impairment and early Alzheimer's disease: A Pilot study. J Alzheimers Dis 2017;57:705-10.

6. De Guio F, Vignaud A, Ropele S, Duering M, Duchesnay E, Chabriat $\mathrm{H}$, et al. Loss of venous integrity in cerebral small vessel disease: a 7-T MRI study in cerebral autosomal-dominant arteriopathy with subcortical infarcts and leukoencephalopathy (CADASIL). Stroke 2014;45:2124-6.

7. Shaaban CE, Aizenstein HJ, Jorgensen DR, MacCloud RL, 
Meckes NA, Erickson KI, et al. In vivo imaging of venous side cerebral small-vessel disease in older adults: An MRI method at 7T. AJNR Am J Neuroradiol 2017;38:1923-8.

8. Dalton SR, Fillman EP, Ferringer T, Tyler W, Elston DM. Smooth muscle pattern is more reliable than the presence or absence of an internal elastic lamina in distinguishing an artery from a vein. J Cutan Pathol 2006;33:216-9.

9. Keith J, Gao FQ, Noor R, Kiss A, Balasubramaniam G, Au K, et al. Collagenosis of the deep medullary veins: An underrecognized pathologic correlate of white matter hyperintensities and periventricular infarction? J Neuropathol Exp Neurol 2017;76:299-312.

10. Pantoni L. Cerebral small vessel disease: from pathogenesis and clinical characteristics to therapeutic challenges. Lancet Neurol 2010;9:689-701.

11. Thomas JM, Surendran S, Abraham M, Sasankan D, Bhaadri $\mathrm{S}$, Rajavelu A, et al. Gene expression analysis of nidus of cerebral arteriovenous malformations reveals vascular structures with deficient differentiation and maturation. PLoS One 2018; $13: \mathrm{e} 0198617$.

12. Vanlandewijck M, He L, Mäe MA, Andrae J, Ando K, Del Gaudio F, et al. A molecular atlas of cell types and zonation in the brain vasculature. Nature 2018;554:475-80.

13. Qiu W, Zhang H, Bao A, Zhu K, Huang Y, Yan X, et al. Standardized operational protocol for human brain banking in China. Neurosci Bull 2019;35:270-6.
14. Samarasekera N, Al-Shahi Salman R, Huitinga I, Klioueva N, McLean CA, Kretzschmar H, et al. Brain banking for neurological disorders. Lancet Neurol 2013;12:1096-105.

15. Betts JG, Young KA, Wise JA, Johnson E, Poe B, Kruse DH, et al. Anatomy and Physiology II. Houston: OpenStax; 2013.

16. Halestrap AP. The monocarboxylate transporter family-Structure and functional characterization. IUBMB Life 2012;64:1-9.

17. Kishimoto A, Takahashi-Iwanaga H, Watanabe MM, Iwanaga T. Differential expression of endothelial nutrient transporters (MCT1 and GLUT1) in the developing eyes of mice. Exp Eye Res 2016;153:170-7.

18. Iwanaga T, Kishimoto A. Cellular distributions of monocarboxylate transporters: a review. Biomed Res 2015;36:279-301.

19. Nehlig A, Pereira de Vasconcelos A. Glucose and ketone body utilization by the brain of neonatal rats. Prog Neurobiol 1993;40:163-221.

20. Chiry O, Pellerin L, Monnet-Tschudi F, Fishbein WN, Merezhinskaya N, Magistretti PJ, et al. Expression of the monocarboxylate transporter MCT1 in the adult human brain cortex. Brain Res 2006;1070:65-70.

21. Kovacs L, Cao Y, Han W, Meadows L, Kovacs-Kasa A, Kondrikov D, et al. PFKFB3 in smooth muscle promotes vascular remodeling in pulmonary arterial hypertension. Am J Respir Crit Care Med 2019;200:617-27.

Received for publication: 15 July 2021. Accepted for publication: 8 September 2021.

This work is licensed under a Creative Commons Attribution-NonCommercial 4.0 International License (CC BY-NC 4.0).

(C) Copyright: the Author(s), 2021

Licensee PAGEPress, Italy

European Journal of Histochemistry 2021; 65(s1):3306

doi:10.4081/ejh.2021.3306 